\title{
QUASAR ABSORPTION LINE OBSERVATIONS
}

\author{
JILL BECHTOLD \\ Steward Observatory, University of Arizona \\ Tucson, AZ 85721, USA
}

\begin{abstract}
Searches for molecular gas in high redshift quasar absorptionline galaxies are reviewed. Despite a great deal of effort, firm evidence has been found in only a few, atypical cases - in clouds which are very close to a luminous quasar, or in objects which have been magnified by gravitational lensing. Recent observations suggest that normal galaxies at $z \sim 3$ had starformation rates about ten times that of the present-day Milky Way. Hence, detections of molecular gas by means of the millimeter transitions of $\mathrm{CO}$ will require more sensitive observations than have been attempted to date.
\end{abstract}

\section{Introduction}

Many of the narrow absorption lines seen in the ultraviolet spectra of high redshift quasars originate in the interstellar gas of intervening galaxies. These quasar absorbers have provided a wealth of detailed information about the formation and evolution of normal galaxies at early epochs, and have been used to address many questions of cosmological interest. How exactly were galaxies assembled, and what did the gaseous precursors to galaxies look like? Can quasar absorbers be used to trace the chemical evolution history of galaxies? What was star-formation like at high redshift, and how did the formation of the first generation of stars proceed? Can we identify objects which can be described as protogalaxies, that is, galaxies forming their first generation of stars? How are the quasar absorbers related to the development of active nuclei, the evolution of the intergalactic medium, and the formation of clusters of galaxies?

Much effort has gone into detecting the molecular gas in quasar absorber galaxies at high redshifts, with limited success. In principle, spectroscopy of molecules in quasar absorbers will provide a sensitive set of diagnostics for evaluating quantities such as the radiation field, and can complement 
spectroscopy of atomic transitions. More importantly, a complete understanding of the history of star-formation in galaxies must include measures of the molecular gas from which the stars form.

For a general review of the field of quasar absorption lines, the reader is referred to the articles included in Meylan (1995); molecular gas in high redshift galaxies is also reviewed by Radford $(1996 \mathrm{a}, \mathrm{b})$.

\section{2. $z<1-\mathrm{Mg}$ II absorbers}

For redshifts $z \sim 0-1$, corresponding to look-back times of 5-10 Gyrs, Mg II $\lambda \lambda 2796,2803$ absorbers provide a sample of gas-rich galaxies for molecular searches (see Steidel \& Sargent 1992; Aldcroft, Bechtold \& Elvis 1994 and references therein; for catalogs of quasar absorbers, see York et al. 1991, 1996; Junkkarinen, Hewitt \& Burbidge 1991). The galaxies causing absorption have been identified for most systems searched (Bergeron \& Boissé 1991, Bergeron, Cristiani \& Shaver 1992; Steidel 1993; Steidel, Dickinson \& Persson 1994; Steidel 1995). The galaxies have colors like normal Sb galaxies on average, and a wide range of luminosity at every redshift (Steidel, Dickinson \& Persson 1994). The lack of strong evolution in the galaxy colors indicates that the star-formation rate in the $\mathrm{Mg}$ II absorber galaxies is fairly constant over this redshift range. The galaxies typically show signs of on-going star-formation, such as strong [O II] $\lambda 3727$ emission (Yanny, York \& Williams 1990; Yanny 1990; Bergeron \& Boissé 1991; Yanny \& York 1992; Nelson \& Malkan 1992).

$\mathrm{CO}$ millimeter emission has been difficult to detect for most $\mathrm{Mg}$ II absorbers. For single dish telescopes (e.g. the NRAO $12 \mathrm{~m}$ or CSO), the typical limits reached in several hours of exposure allow one to detect a galaxy as bright as the Milky Way out to redshifts of $\mathrm{z} \sim 0.1$, and a luminous starforming galaxy like Arp 220 to $z \sim 0.4$. The problem is that the beam size ( 1-2 arcminutes) is large compared to the size of the galaxies, at all but the smallest redshifts. There are only a handful of detections for the most nearby absorbers (Walker, Bechtold \& Black 1995), with the notable exception of the $z=0.49$ absorber of the $z=0.87$ quasar 3C196 (Frayer \& Brown 1995). The mass for this galaxy in molecular $\mathrm{H}_{2}$ (given the standard $\mathrm{CO}$ to $\mathrm{H}_{2}$ ratio, and other assumptions), is $\sim 10^{11} \mathrm{~h}_{75}{ }^{-2} \mathrm{M}_{\odot}\left(\mathrm{q}_{0}=0.5\right)$, much greater than the Milky Way. Since most Mg II absorber galaxies are inferred from their colors to have modest star-formation rates, it is perhaps not surprising that most have not been detected to date.

A very promising approach has been pursued by Wiklind \& Combes (1994b, 1995a, 1996), who have detected millimeter-wave absorption by molecules in three objects at $z=0.24,0.68$ and 0.88 . Since absorption is very sensitive to small columns of molecular gas (compared to emission), 
they have been able to detect rare molecules such as $\mathrm{HCO}^{+}, \mathrm{HCN}, \mathrm{HNC}$ and $\mathrm{N}_{2} \mathrm{H}^{+}$; formaldehyde has been detected by Menten \& Reid (1996) for the $z=0.68$ system. The relative abundances of the various species are similar to those found in the Milky Way. It is relevant to note that the objects which have detected millimeter absorption are very red compared to the quasars used in optical/UV absorption-line studies, probably indicating that the sight-lines are heavily reddened by dust. In fact, around a dozen quasar absorption line systems have been searched for millimeter absorption by both Wiklind \& Combes (1995b) and others (Takahara et al. 1984, 1987; Douglas et al. 1992) with no success.

\section{3. $z>2$ - Damped Lyman Alpha absorbers}

Damped Ly $\alpha$ absorbers are identified by means of their strong Ly $\alpha$ absorption, which by definition shows damping wings in moderate dispersion optical spectra (Wolfe et al. 1986; Lanzetta et al. 1991; Wolfe et al. 1995 and references therein). These absorbers have spectra which are reminiscent of diffuse interstellar clouds in the Milky Way, and, at $z>2$ (corresponding to look-back times of 10-20 Gyrs), have been suggested as the progenitors of galactic disks or halos (Wolfe et al. 1994 and references therein). The damping wings of the Ly $\alpha$ profile allow an accurate determination of the neutral hydrogen column density, $\mathrm{N}(\mathrm{HI})$, and the detections of weak UV absorption lines allow accurate abundance determinations (Wolfe et al. 1986; Lanzetta et al. 1991; Lu \& Wolfe 1994; Wolfe et al. 1995, Pettini et al. 1995; Meyer, Lanzetta \& Wolfe 1995; Lu et al. 1996 and references therein). Pettini et al. (1995) find that out to $z \sim 3.5$ metal-line absorption is always seen, with metallicities $0.1-0.01$ solar. The total amount of mass in gas in the damped Ly $\alpha$ absorbers is very large at $z \sim 3$, comparable to the amount of mass in stars today (Lanzetta et al. 1995; Wolfe 1995). Although there are still a "factor of a few" uncertainty associated with this statement, it suggests that in fact we are seeing galaxies at $\mathrm{z} \sim 3$ in which a majority of the baryons are in gas, and most of the stars have not yet formed. Measuring the molecular gas in these systems is clearly important for understanding the history of star-formation in normal galaxies.

The first efforts to detect molecular gas in damped Ly $\alpha$ absorbers used the Lyman and Werner UV absorption transitions of $\mathrm{H}_{2}$; the work to date is summarized in Table 1. Since the lines are redshifted into the optical, high resolution spectroscopy is possible from the ground. The searches have been mostly negative, and sensitive limits have been obtained. The limits on the fraction of hydrogen in molecular form, $f\left(\mathrm{H}_{2}\right)=2 N\left(\mathrm{H}_{2}\right) / N(\mathrm{H})$, are extremely low compared to diffuse interstellar clouds in the Milky Way. This has been interpreted as indicating a very low dust-to-gas ratio and/or 
a very high UV photon to gas density ratio in the typical absorber. The only confirmed detection at the time of the conference (however see $\mathrm{Ge}$ \& Bechtold 1996b) is the $\mathrm{z}=2.85$ absorber of $0528-25$, which is probably a special case. This absorber has a redshift greater than the quasar emission redshift, and while there is substantial evidence that the absorbing material is located well outside the central engine of the quasar (Moller \& Warren 1993), the proximity to a very luminous, radio-loud quasar makes this system potentially atypical.

TABLE 1. Searches for $\mathrm{H}_{2}$ Absorption in Damped Ly $\alpha$ Systems

\begin{tabular}{|c|c|c|c|c|c|}
\hline QSO & $z_{a b s}$ & $z_{e m}$ & $N(\mathrm{H} \mathrm{I})$ & $f_{\mathrm{H}_{2}}$ & Refn. \\
\hline $0100+13$ & 2.30 & 2.68 & $2.5 \times 10^{21} \mathrm{~cm}^{-2}$ & $<4 \times 10^{-6}$ & 1 \\
\hline $1337+11$ & 2.79 & 2.92 & $8.0 \times 10^{20} \mathrm{~cm}^{-2}$ & $<1.3 \times 10^{-4}$ & 2 \\
\hline $0528-25$ & 2.81 & 2.77 & $2.0 \times 10^{21} \mathrm{~cm}^{-2}$ & $10^{-3}$ & 3 \\
\hline $0000-26$ & 3.39 & 4.11 & $2.0 \times 10^{21} \mathrm{~cm}^{-2}$ & $<3 \times 10^{-6}$ & 4 \\
\hline $1033-03$ & 4.17 & 4.50 & $1.5 \times 10^{20} \mathrm{~cm}^{-2}$ & $<1.1 \times 10^{-5}$ & 5 \\
\hline
\end{tabular}

References - (1) Black, Chaffee \& Foltz (1987). (2) Lanzetta et al. (1989). (3) Foltz, Chaffee, \& Black (1988). (4) Levshakov et al. (1992). (5) Ge \& Bechtold (1996a).

After extremely bright CO emission was detected for the $z=2.3$ IRAS source F10214+4724 (Brown \& Vanden Bout 1991; Solomon et al. 1992; Tsuboi \& Nakai 1992), several groups began searching for $\mathrm{CO}$ and other millimeter-wave emission from damped Ly $\alpha$ absorbers, as well as other objects (quasars, radio galaxies, protogalaxy candidates) at comparable redshift (Wiklind \& Combes 1994a; Frayer, Brown \& Vanden Bout 1994a; Barvainis et al. 1994; Walker, Bechtold \& Black 1995; Yun \& Scoville 1995; Evans et al. 1996). Note that searches for molecular emission complement the $\mathrm{H}_{2}$ absorption studies described above: it could be that the quasars pick out lines-of-sight through the foreground galaxy which have unusually low reddening, and a large mass of molecular gas and dust is present, offset from the quasar line-of-sight. Despite early optimism, most searches for molecular emission from damped Ly $\alpha$ absorbers, however, have been unsuccessful, and, as of this writing, there is only a weak detection of $\mathrm{CO}$ for the $z=3.1$ damped Ly $\alpha$ absorber of PC 1643+4631A (Frayer, Brown \& Vanden Bout 1994b). The extensive efforts turned out to be mostly an education in base-line ripple and back-end stability, since these observations push the single-dish receivers to low noise levels not normally attempted. The detection reported by Brown \& Vanden Bout (1993), for example, was later not confirmed by Wiklind \& Combes (1994a). Subsequently, IRAS F10214+4724 turned out to be magnified by gravitational lensing (Eisen- 
hardt et al. 1996, and references therein), and the other confirmed $z \sim 2$ detection, the "Cloverleaf" broad absorption line QSO (Barvainis et al. 1994) at $z=2.5$ is also lensed and probably magnified by about a factor of 10 (Kayser et al. 1990). At this conference, we heard an exciting report of the detection of $\mathrm{CO}$ at $z=4.69$ by Omont (Omont et al. 1996; see also Ohta et al. 1996) from the radio-quiet quasar BR1202-0725. Whether this system is typical of high redshift quasars is not clear: Barvainis \& Antonuci (1996) have used the VLA to search for molecular emission from a number of $z \sim 4$ quasars, and have reported no detections, but with slightly less sensitive limits than the BR 1202-0725 result.

In any event, the confirmed detections of millimeter molecular emission to date have been by and large in objects magnified by lensing, or in objects associated with AGN activity, never "normal" galaxies or proto-galaxies which might be representative of the predecesors of common galaxies like the Milky Way. The reader is referred to the figure in Walker, Bechtold \& Black (1995) for a summary of the results for damped Ly $\alpha$ absorbers and Mg II absorbers.

The difficulty in detecting molecular masses for the damped Ly $\alpha$ absorbers can be understood in light of new measurements of star-formation rates in the associated galaxies. The classical technique for estimating the global star-formation rates of galaxies is to observe their nebular emission lines, which are produced in H II regions excited by young stars (Kennicutt 1983). For damped Ly $\alpha$ absorbers, a great deal of effort has been devoted to searching for nebular Ly $\alpha$ emission, through optical long-slit spectroscopy, narrow-band imaging, and Fabry-Perot imaging (Lowenthal et al. 1991, 1995 and references therein; Wolfe et al. 1992). With few exceptions, Ly $\alpha$ emission is not detected, and the inferred limits on star-formation rate are low, typically less than $\sim 0.5 \mathrm{M}_{\odot} \mathrm{yr}^{-1}$. However, the small amount of dust inferred to be present in damped Ly $\alpha$ absorbers (Pei, Fall \& Bechtold 1991; Pettini et al. 1995 and references therein), can easily extinguish the Ly $\alpha$ emission (Charlot \& Fall 1991, 1993; Valls-Gabaud 1993), and the observations are consistent with star-formation rates of up to $1000 \mathrm{M}_{\odot} \mathrm{yr}^{-1}$, given plausible assumptions for the amount and distribution of the dust.

$\mathrm{H} \alpha$ is less affected by dust, but for the $\mathrm{z}>2$ damped Ly $\alpha$ systems, $\mathrm{H} \alpha$ is redshifted into the near-IR. Searches are therefore less sensitive, but the rapid improvement in IR narrow-band imaging capability has just recently made these searches feasible with tunable narrow pass-band filters (Elston et al. 1991, 1996; Bunker et al. 1995). The typical star-formation rates are $5-20 \mathrm{M}_{\odot} \mathrm{yr}^{-1}$, and the emission is generally compact, and displaced several arcseconds from the quasar on the sky. For some cases where $\mathrm{H} \alpha$ is detected, Ly $\alpha$ is weak or not detected, at many factors below Case B predictions (Elston et al. 1991, 1996; Bunker et al. 1995; Deharveng, Buat 
\& Bergeron 1995; Djorgovski et al. 1996). In these cases, dust obscuration may be important.

If the typical star-formation rate in the damped Ly $\alpha$ absorbers is $\sim 10$ $\mathrm{M}_{\odot} \mathrm{yr}^{-1}$ then the lack of success in detecting millimeter-wave $\mathrm{CO}$ is not surprising. A factor of $\sim 10-50$ improved sensitivity over the single dish measurements will be required; detections should be possible with current and planned interferometers.

\section{4. $\mathrm{MS1512-cB58}$}

Recently, a number of high redshift galaxies have been found which appear to be comprised primarily of very young stars, with rest UV spectra similar to those of local star-burst galaxies, and inferred star-formation rates of $>10 \mathrm{M}_{\odot} \mathrm{yr}^{-1}$ (Malkan, Teplitz \& McLean 1995; Steidel et al. 1996; Yee et al. 1996b; Ebbels et al. 1996). The ubiquity of these galaxies at $z \sim 3$ suggests that they may be the progenitors of common galaxies like the Milky Way (Steidel et al. 1996), and their properties indicate that they may be closely related, if not identical, to the galaxies associated with damped Ly $\alpha$ absorbers. By far the brightest example, MS1512-cB58, was discovered serendipitously in the CNOC1 redshift survey (Yee, Ellingson \& Carlberg 1996a) and has an optical/IR spectral energy distribution which is well fit by a continuous star-formation model with an age of only 10-20 million years, with reddening of $E(B-V) \sim 0.3$ (Yee et al. 1996; Ellingson et al. 1996). The observered luminosity of the stellar continuum at $1500 \AA$ implies an apparent star-formation rate of $4700 \mathrm{M}_{\odot} \mathrm{yr}^{-1}$, neglecting the possible magnification by lensing (see Ellingson et al. 1996, Williams \& Lewis 1996, for a discussion of the effect of lensing).

These properties suggested that MS1512-cB58 would be a good candidate for a millimeter-wave search for $\mathrm{CO}(3-2)$ emission, which, at $z=2.7$, is redshifted to $92.87 \mathrm{GHz}$. Deep observations were obtained at the NRAO $12 \mathrm{~m}$ and OVRO six element interferometer early this year, and are reported by Frayer et al. (1996). No emission was detected, with an upper limit to the mass of $\mathrm{H}_{2}$ of $2.6 \times 10^{10} \mathrm{~h}_{75}{ }^{-1} \mathrm{M}_{\odot}\left(\mathrm{q}_{0}=0.5\right)$. If the star-formation rate is $4700 \mathrm{M}_{\odot} \mathrm{yr}^{-1}$, then the ratio of star-formation rate to mass in molecular gas, a measure of the star-formation efficiency, is $>180 \mathrm{Gyr}^{-1}$, independent of the unknown amount of magnification by lensing. By contrast, local starbursts have values of $1-10 \mathrm{Gyr}^{-1}$. Bechtold et al. (1996) subsequently measured the $\mathrm{H} \alpha$ emission from MS1512-cB58, and derived a star-formation rate somewhat lower than that derived from the UV continuum, $270 \mathrm{M}_{\odot}$ $\mathrm{yr}^{-1}$. A number of effects can cause the UV continuum to overestimate the star-formation rate in star-burst galaxies (Meuer et al. 1995; Bechtold et al. 1996), but even if this lower star-formation rate is more reliable, MS1512- 
cB58 is a remarkable galaxy, which is manufacturing stars with relatively high efficiency, given its apparently small molecular mass.

\section{Summary}

A picture is emerging of the history of star-formation in galaxies from the time of their formation up to the present day. For $z<1$, the population of galaxies probed appears to be slowly evolving, with modest star-formation rates. Reddened lines-of sight have yielded rich molecular absorption spectra in the millimeter, but otherwise, millimeter-wave emission and absorption have been difficult to detect. For $z>2$, the damped Ly $\alpha$ absorbers and recently discovered $z \sim 3$ star-burst galaxies may have star-formation rates $\sim 10 \mathrm{M}_{\odot} \mathrm{yr}^{-1}$, and be the progenitors of normal galaxies like the Milky Way. However, evidence for molecular gas, in either emission or absorption, has not yet been found for most of them. In at least one case, the lack of detectable $\mathrm{CO}$ emission may imply a high efficiency of star-formation, compared to local starbursts and star-formation regions in the Milky Way. Studies of molecular gas in galaxies identified in quasar absorption-line surveys will continue to be an important set of observations necessary to complete our understanding of star-formation in the early Universe.

\section{Acknowledgements}

I am grateful for the contributions of collaborators Jian Ge, Dave Frayer, Richard Elston, Howard Yee, John Black and Connie Walker. Data reported here were obtained at the MMT, NRAO 12m, OVRO, IRTF, CSO and JCMT, and the contributions of the staffs of these observatories is greatly appreciated. Financial support was provided by NSF AST-9058510.

\section{References}

Aldcroft, T. L., Bechtold, J., Elvis, M., 1994, ApJS, 93, 1.

Barvainis, R., Tacconi, L., Antonucci, R., Alloin, D., Coleman, P., 1994, Nature, 371, 586.

Barvainis, R., Antonucci, R., 1996, PASP, 108, 187.

Bechtold, J., Yee, H. K. C., Elston, R., Ellingson, E., 1996, AJ, submitted.

Bergeron, J., Boisse, P. 1991, A\&A, 243, 344

Bergeron, J., Cristiani, S., Shaver, P. 1992, A\&A, 257, 417

Black, J. H., Chaffee, F. H., Foltz, C. B., 1987, ApJ, 317, 442.

Brown, R. L., Vanden Bout, P. A., 1991, AJ, 102, 1956.

Brown, R. L., Vanden Bout, P. A., 1993, ApJ, 412, L21.

Bunker, A. J., Warren, S. J., Hewett, P. C., Clements, D. L. 1995, MNRAS, 273, 513

Charlot, S. \& Fall, S. M. 1991, ApJ, 378, 471

Charlot, S. \& Fall, S. M. 1993, ApJ, 415, 580

Deharveng, J. M., Buat, V., Bergeron, J. 1995, A\&A, 298, 57

Djorgovski, S. G., Pahre, M. A., Bechtold, J., Elston, R. 1996, Nature, 382, 234 
Douglas, N. G., Radford, S. J. E., Roland, J., Webb, J. K., 1992, A\&A, 262, 8.

Ebbels, T.M.D., LeBorgne, J.-F., Pelio, R., Ellis, R. S., Kneib, J.-P., Smail, L., Sanahuja, B., 1996, MNRAS, submitted.

Eisenhardt, P. R., Armus, L., Hogg, D. W., Soifer, B. T., Neugebauer, G., Werner, M. W., 1996, ApJ, 461, 72.

Ellingson, E., Yee, H. K. C., Bechtold, J., Elston, R., 1996, ApJ, 466, L71.

Elston, R., Bechtold, J., Lowenthal, J., Rieke, M. 1991, ApJ Letters, 373, L39

Elston, R., Bechtold, J., Cutri, R., 1996, in preparation.

Evans, A. S., Sanders, D. B., Mazzarella, J. M., Solomon, P. M., Downes, D., Kramer, C., Radford, S. J. E., 1996, ApJ, 457, 658.

Foltz, C. B., Chaffee, F. H., Black, J. H., 1988, ApJ, 324, 267.

Frayer, D. T., Brown, R. L., 1995, in QSO Absorption Lines, Proceedings of the ESO Workshop Held at Garching, Germany, ed. G. Meylan, (Berlin:Springer), p. 179.

Frayer, D. T., Brown, R. L., Vanden Bout, P. A., 1994a, BAAS, 185, 112.01.

Frayer, D. T., Brown, R. L., Vanden Bout, P. A., 1994b, ApJ Letters, 433, L5.

Frayer, D. T., Papadopoulous, P. P., Bechtold, J., Yee, H. K. C., Seaquist, E. R., Scoville, N. Z., 1996, ApJ Letters, submitted.

Ge, J., Bechtold, J., 1996a, ApJ, submitted.

Ge, J., Bechtold, J., 1996b, ApJ Letters, submitted.

Junkkarinen, V., Hewitt, A., Burbidge, G., 1991, ApJS, 77, 203.

Kayser, R., Surdej, S., Condon, J. J., Kellerman, K., Magain, P., Remy, M., Smette, A., 1990, ApJ, 364, 15.

Kennicutt, R. C., Jr., 1983, ApJ, 272, 54

Lanzetta, K. M., Wolfe, A. M., Turnshek, D. A., 1989, ApJ, 344, 277.

Lanzetta, K. M., McMahon, R. G., Wolfe, A. M., Turnshek, D. A., Hazard, C., Lu, L. 1991, ApJS, 77, 1

Lanzetta, K. M., Wolfe, A. M., Turnshek, D. A. 1995, ApJ, 440, 435

Levshakov, S. A., Chaffee, F. H., Black, J. H., 1992, A\&A, 262, 385.

Lowenthal, J. D., Hogan, C. J., Green, R. F., Caulet, A., Woodgate, B. E., Brown, L., Foltz, C. B. 1991, ApJ, 377, L73

Lowenthal, J. D., Hogan, C. J., Green, R. F., Woodgate, B., Caulet, A., Brown, L., Bechtold, J. 1995, ApJ, 451, 484

Lu, L., Wolfe, A. M. 1994, AJ, 108, 44

$\mathrm{Lu}$, L. et al., 1996, preprint

Malkan, M., Teplitz, H., McLean, I., 1995, ApJ Letters, L5.

Menten, K. M., Reid, M. J., 1996, ApJ 465, L99.

Meurer, G. R., Heckman, T. M., Leitherer, C., Kinney, A., Robert, C., Garnett, D. R., 1995, AJ, 110, 2665.

Meyer, D. M., Lanzetta, K. M., Wolfe, A. M., 1995, ApJ Letters, 451, L13.

Meylan, G. 1995, QSO Absorption Lines: Proceedings of the ESO Workshop Held at Garching, Germany, 21-24 November 1994 (Berlin:Springer)

Moller, P., Warren, S. J., 1993, A\&A, 270, 43.

Nelson, B. O., Malkan, M. A., 1992, ApJS, 82, 447.

Ohta, K., Yamada, T., Nakanishi, K., Kohno, K., Akiyama, M., Kawabe, R., 1996, Nature, $382,426$.

Omont, A., Petitjean, P., Guilloteau, S., McMahon, R. G., Solomon, P. M., Pecontal, E., 1996, Nature, 382, 428.

Pei, Y. C., Fall, S. M., Bechtold, J. 1991, ApJ, 378, 6

Pettini, M., Lipman, K., Hunstead, R. W., 1995, ApJ, 451, 100.

Radford, S. J. E., 1996a, in Cold Gas at High Redshift, eds. M. Bremer, H. Röttgering, P. van der Werf, C.L. Carilli, (Dordrecht: Kluwer).

Radford, S. J. E., 1996b, in IAU Symp. 170: CO: Twenty-five Years of Millimeter-wave Spectroscopy, eds. W.B. Latter, S.J.E. Radford, P.R. Jewell, J.G. Mangum, J. Bally, (Dordrecht:Kluwer) in press.

Solomon, P. M., Radford, S. J. E., Downes, D., 1992, Nature, 356, 318. 
Steidel, C. C. 1993, in Proceedings of the Third Teton Summer School on The Environment and Evolution of Galaxies, July 1992, eds. J. M. Shull \& H. A. Thronson, (Dordrecht:Reidel), p. 263

Steidel, C. C. 1995, in QSO Absorption Lines: Proceedings of the ESO Workshop Held at Garching, Germany, ed. G. Meylan, (Berlin:Springer) p. 139

Steidel, C. C., Sargent, W. L. W., 1992, ApJS, 80, 1.

Steidel, C. C., Dickinson, M., Persson, S. E. 1994, ApJ Letters, 437, L75

Steidel, C. C., Giavalisco, M., Pettini, M., Dickinson, M., Adelberger, K. L., 1996, ApJ Letters, 462, L17

Takahara, F., Sofue, Y., Inou, M., Nakai, N., Tabara, H., Kato, T., 1984, PASJ, 36, 387.

Takahara, F., Nakai, N., Briggs, F. H., Wolfe, A. M., Liszt, H. S., 1987, PASJ, 39, 933.

Tsuboi, M., Nakai, N., 1992, PASJ, 44, L241.

Valls-Gabaud, D., 1993, ApJ, 419, 7

Walker, C. E., Bechtold, J., Black, J. H., 1995, Poster contribution to IAU Symposium 170, CO: Twenty-five Years of Millimeter-wave Spectroscopy, ed. W. Latter et al.

Wiklind, T., Combes, F., 1994a, A\&A, 288, L41.

Wiklind, T., Combes, F., 1994b, A\&A, 286, L9.

Wiklind, T., Combes, F., 1995a, A\&A, 299, 382.

Wiklind, T., Combes, F., 1995b, in QSO Absorption Lines:, Proceedings of the ESO Workshop Held at Garching, Germany, ed. G. Meylan, (Berlin:Springer), p. 175.

Wiklind, T., Combes, F., 1996, Nature, 379, 139.

Williams, L. L. R., Lewis, G. F., 1996, MNRAS, in press.

Wolfe, A. M. 1995, in QSO Absorption Lines: Proceedings of the ESO Workshop Held at Garching, Germany, ed. G. Meylan, (Berlin:Springer) p. 13

Wolfe, A. M., Turnshek, D. A., Smith, H. E., Cohen, R. D. 1986, ApJS, 61, 249

Wolfe, A. M., Lanzetta, K. M., Turnshek, D. A., Oke, J. B. 1992, ApJ, 385, 151

Wolfe, A. M., Fan, X., Tytler, D., Vogt, S. S., Keane, M. J., Lanzetta, K. M. 1994, ApJ Letters, 435, L101

Wolfe, A. M., Lanzetta, K. M., Foltz, C. B., Chaffee, F. H. 1995, ApJ, 454, 698

Yanny, B. 1990, ApJ, 351, 396

Yanny, B., York, D. G., Williams, T. B. 1990, ApJ, 351, 377

Yanny, B., York, D. G. 1992, ApJ, 391, 569

Yee, H. K. C., Ellingson, E., Carlberg, R. G. 1996a, ApJS, 102,

Yee, H. K. C., Ellingson, E., Bechtold, J., Carlberg, R. G., Cuillandre, J.C. 1996b, AJ, $111,1783$.

York, D. G., Yanny, B., Crotts, A., Carilli, C., Garrison, E., Matheson, L., 1991, MNRAS, $250,24$.

York, D. G., et al., 1996, in preparation.

Yun, M. S., Scoville, N., 1996, in IAU Symp. 170: CO: Twenty-five Years of Millimeterwave Spectroscopy, ed. W.B. Latter, S.J.E. Radford, P.R. Jewell, J.G. Mangum, J. Bally, (Dordrecht:Kluwer) in press.

\section{Discussion}

Irvine: Could you not have a lot of $\mathrm{H}_{2}$ in MS1512-cB58, provided that the fractional abundance, $\mathrm{CO} / \mathrm{H}_{2}$, were much less than that in local galaxies?

Bechtold: Yes, in principle - the $\mathrm{CO}$ to $\mathrm{H}_{2}$ conversion factor is expected to vary with metallicity and physical conditions. However, we estimate the metallicity of MS1512-cB58 from its extinction and nebular line ratios to be about $0.2-0.6$ solar. The strong metal lines in its rest UV spectrum also suggest that the metallicity is high. If this is the case, the Galactic $\mathrm{CO}$ to $\mathrm{H}_{2}$ conversion factor underestimates the molecular gas mass by only about a factor of 2 , and the qualitative results remain unchanged. 
国 